una lunghezza totale di circa $10000 \mathrm{~km}$. All'ovest del Ticino i più importanti sono: il Canale Cavour; il Canale Elena, inaugurato nel 1954, che conduce a quello l'acqua del Ticino; e tutti gli altri canali statali piemontesi (Canali Demaniali). All'est del Ticino i canali: Villoresi, Naviglio Grande, Martesana, Pavia, Muzza e quello industriale di Vizzola. I laghi alpini sono stivati da dighe regolatrici per aumentare la massa d'acqua da mettere a disposizione. I più grandi canali scorrono parelleli alle Alpi e al $\mathrm{Po}$, da un affluente all'altro, cosicchè la parte della pianura verso il Po può essere quasi completamente irrigata. Là, dove i canali passano su terreno ciottoloso, devono essere murati per impedire grandi perdite d'acqua. Sopratutti, il canale Villoresi, che scorre nell'asciutta pianura padana al Nord di Milano, è uno di questi. Gli interessati all'irrigazione sono, nella zona di cui abbiamo parlato, riuniti in tre grandi società (Associazione d'irrigazione Ovest e Est Sesia, Consorzio Villoresi), che provvedono alla ripartizione dell'acqua, al mantenimento e al miglioramento dei canali. Anche per le acque del 'Ticino e dell'Adda sono stati creati speciali Consorzi. Le maggiori colture d'irrigazione sono le risaie e le marcite.

\title{
MANNHEIM - LUDWIGSHAFEN
}

\section{Gerhard Ammann}

Wer in der Oberrheinischen Tiefebene nordwärts fährt, wird häufig und zu jeder Jahreszeit nördlich von Karlsruhe, etwa auf der Höhe von Speyer, cine zunehmende Trübung der Luft feststellen können. Es ist dies das erste Anzeichen, daß man sich einem hochindustrialisierten Gebiet nähert, nämlich dem Raume Mannheim-Ludwigshafen. Betreten wir dann eine der beiden Städte, so stellen wir nicht nur nebelartigen, oft sehr dichten Dunst fest, sondern auch einen beißenden Geruch, der auf Abgase chemischer Industrie schließen läßt. Es liegt eine Industriezusammenballung vor uns, wie sie Süddeutschland in dieser Größe und Ausprägung sonst nicht mehr kennt. Ihre Entstehung, Entwicklung und heutige Bedeutung soll im Folgenden darzustellen versucht werden.

\section{LAGE，NATÜRLICHE UND HISTORISCHE VORAUSSETZUNGEN}

Der nördliche Teil der Oberrheinischen Tiefebene, in dem Mannheim und Ludwigshafen liegen, wird im $\mathrm{W}$ durch die Haardt oder den Pfälzer Wald und im E durch den Odenwald (N) und den Schwarzwald (S) begrenzt. Zwischen Odenwald und Schwarzwald schiebt sich ein $50 \mathrm{~km}$ breites, sanft nach $\mathrm{E}$ ansteigendes Hügelland ein, der Kraichgau, auch Kraichgaulücke genannt. Sie stellt die ideale Pforte für den Verkehr von $E$ in die Tiefebene oder von $W$ nach dem mittleren Neckar, dann nach Franken und Bayern dar.

Die beiden Städte liegen nur noch $95 \mathrm{~m}$ ü. M. an und gegenüber der Neckarmündung, zu beiden Seiten des Rheines. Sie befinden sich nicht innerhalb der überschwemmungssicheren Zone auf den Hochufern (mehrere Meter hohe Erosionsstufe), sondern außerhalb derselben in der Flußniederung (Rheinaue). Zwischen den beiden Hochufern mäandrierte der Rhein sehr stark und überschwemmte bei Hochwasser regelmäßig große Gebiete. Auch der Neckar veränderte seinen Lauf in historischer Zeit noch wesentlich. So nimmt man an, daß die Neckarmündung im Frühmittelalter noch südlich der heutigen Stadt Mannheim lag.

Da im Mittelalter in der Rheinaue wegen dieser dauernden Überschwemmungsgefahr Siedlungen von Bedeutung nicht entstehen konnten, entwickelten sich auch Mannheim und Ludwigshafen erst in der Neuzeit.

Im Raume Mannheim-Ludwigshafen waren Speyer und Worms die wichtigsten Städte links des Rheines. Sie liegen auf dem Hochufer, das dort ursprünglich bis hart an den Fluß vorstieß. Zwischen den erwähnten Städten wurde der Rheinlauf von den Neckarfluten stark nach W gedrängt, und das Hochufer hat sich entsprechend schwach 
entwickelt. Westlich von Ludwigshafen findet sich eine breite Zwischenterrasse, die sogenannte Frankenthaler Terrasse. Sie ersetzt das Hochufer und ist gegenüber der Rheinaue nur wenig abgehoben. Auch sie war früher nicht überschwemmungssicher, da von den Bächen der Haardt Gefahr drohte und war folglich auch schwach besiedelt. ${ }^{1}$

Zwischen der Frankenthaler Terrasse und dem Gebirge breitete sich ein sehr fruchtbares, flachgewelltes Hügelland aus, das Pfälzer Hügelland. Lößartige Böden ließen hier früh ein Ackerbaugebiet mit großen Dörfern und einer zahlreichen landwirtschaftlichen Bevölkerung entstehen. Die Vorderpfalz, das Gebiet zwischen Rhein und Haardt, besitzt eine der klimatisch begünstigsten Lagen ganz Deutschlands. Früher gab es hier sogar Kastanienwälder. Ein ausgedehnter Weinbau nützt heute noch den Klimavorteil aus. Das Rebgelände ist hier eigenartigerweise zur Hauptsache in der Ebene und im Hügelland zu finden und steigt nicht sehr weit an den Abhängen der Haardt empor.

Nördlich von Breisach besteht am rechten Rheinufer keine mittelalterliche Stadt von Bedeutung, da sich das Hochufer dem Fluß nie genügend nähert. Im Mannheimer Raum besteht der Boden des Hochufers hauptsächlich aus Rheinsanden mit aufgesetzten Binnendünen. Föhrenwälder machen diese Eigenart der Bodenunterlage sichtbar. Der Neckarschwemmfächer mit seinen fruchtbaren, schlickigen Böden stellt als früh besiedeltes Gebiet ein Gegenstück zum Pfälzer Hügelland dar. Am Fuße des Odenwaldes wird unter ähnlichen klimatischen Bedingungen ebenfalls Weinbau betrieben.

So lag mitten im Gebiet der Pfalzgrafen (seit dem 13. Jahrhundert der Kurfürsten von der Pfalz), der Kurpfalz, ein durch die natürlichen Voraussetzungen benachteiligter Raum, der erst spät besiedelt wurde. Er ist begrenzbar durch Speyer im S, Worms im N, sowie durch das Pfälzer Hügelland im $W$ und den Neckarschwemmfächer im E. Er kann als Raum Mannheim-Ludwigshafen bezeichnet werden. Auf den Verkehr wirkte sich dieser Raum als Sperrzone aus. Er zwang die von E durch den Kraichgau heranführenden mittelalterlichen Handelsstraßen nach $\mathrm{N}$ oder $\mathrm{S}$ auszuweichen und den Rheinübergang bei Worms oder Speyer zu suchen. Dort bestanden deshalb Fähren. (Unterhalb Basel gab es ja bis in die Neuzeit keine feste Rheinbrücke.)

Weiter westlich verhinderte die stark bewaldete Haardt, welche von wenigen tiefen, steilwandigen Tälern aufgeschlossen wird, wie eine Barriere das geradlinige Vorstoßen nach Frankreich und erschwerte die Anlage der Straßen, sowie die Abwicklung des Verkehrs sehr. So trafen sich die Straßen von Speyer und Worms erst wieder im Becken von Kaiserslautern.

Die N-S Straßen mieden den Raum Mannheim-Ludwigshafen ebenfalls. Sie folgten im E dem Gebirgsfuß und wichen westlich des Rheins zwischen Speyer und Worms gegen die Haardt aus. Dabei erfolgte im Mittelalter der Hauptverkehr in der Oberrheinebene auf dem linken Rheinufer, wo von Straßburg nordwärts eine ganze Reihe bedeutender Städte lagen (Hagenau, Weißenburg, Landau, Speyer, Worms, Oppenheim, Mainz usw.). Die Straße zwischen Basel und Heidelberg war von geringer Bedeutung. ${ }^{2}$

Die von der Natur gegebenen Nachteile hat der Mensch mit der Zeit zu überwinden gewußt. Vor allem durch die Rheinkorrektion im 19. Jahrhundert ist das Neckarmündungsgebiet voll besiedlungsfähig geworden. Heute ist die ganze Ebene zu beiden Seiten des Rheins ein Gebiet intensiven Ackerbaus mit vielen ertragsreichen, arbeitsintensiven Sonderkulturen. Auf alter Tradition fußend, wird Gemüse- und Obstbau, dann der Anbau von Hopfen und Tabak betrieben. Ein absoluter Höhepunkt findet sich gerade auf den Sandböden mit der Spargelzucht. Weit herum sind die Schwetzinger Spargeln bekannt. Gehäuft liegen große Obst- und Gemüsebörsen. Verbunden mit dem Weinbau am Gebirgsfuß ist hier eine stark besiedelte Agrarlandschaft entstanden, ein gewichtiges Hinterland und Einzugsgebiet für städtische Siedlungen. 


\section{DIE ENTWICKLUNG DER BEIDEN STÄDTE}

Im heutigen Raume Mannheim-Ludwigshafen spielte Heidelberg während Jahrhunderten die große Rolle als Zentrum der Pfalz, genauer gesagt als Residenz des Kurfürstentums Pfalz oder der Kurpfalz.

Das fränkische Fischerdorf Mannheim wird 766 erstmals in einer Urkunde des Klosters Lorsch (etwa $20 \mathrm{~km}$ nordöstlich der Neckarmündung) genannt. Im späteren Mittelalter hatte es einige Bedeutung als Zollstelle für die Rheinschiffahrt; es erreichte damals jedoch die stattliche Größe seiner Nachbardörfer nie.

Zu Beginn des 17. Jahrhunderts begann sich in Deutschland der Dreißigjährige Krieg abzuzeichnen. In der protestantischen Union übernahm der pfälzerische Kurfürst die Führung.

\section{A N N H E I M}

Die Festungsstadt. Parallel zu diesen Vorgängen bahnte sich in Europa ein strategisches Neudenken an, indem man politische Zentren durch die Anlage mächtiger Festungen im Vorgelände derselben zu schützen suchte. ${ }^{3}$ Aus dieser Perspektive gesehen bekam der bisher unbedeutende Mannheimer Raum eine strategisch wichtige Riegelfunktion im Zentrum der Kurpfalz. 1606 wurde auf kurfürstlichen Befehl mit der Anlage einer Wasserfestung im Rhein - Neckar-Mündungswinkel begonnen. Auf zwei Seiten durch Flüsse und im E durch die versumpfte Rheinaue geschützt, deckte sie Heidelberg und die Kraichgaulücke gegen W.

Dieser durch regelmäßige vauban'sche Bastionen geschützten und Friedrichsburg genannten Festung, gliederte sich im NE die Bürgerstadt Mannheim an, die ebenfalls von einem Festungswall umgeben war. Die rechtwinklige Anordnung von Straßenzügen und Häuserblocks hat sich bis auf den heutigen Tag erhalten und bildet ein städtebauliches Charakteristikum der Stadt.

Festung und Stadt mußten zwei Zerstörungen über sich ergehen lassen: Zuerst 1622 durch Tilly, wobei auch die Pfalz verwüstet wurde. Im Zusammenhang mit dem Wiederaufbau legte man auf dem linken Rheinufer die Rheinschanze mit Brückenkopffunktionen an und richtete einen Fährbetrieb ein. Zusammen mit Heidelberg erfuhren 1688 Festung und Stadt während des Orléanschen Erbfolgekrieges eine zweite, diesmal totale Zerstörung.

Die Residenzstadt. Erst 1699 kam es zur dritten und letzten Neugründung. Nachdem die Kurfürsten längere Zeit in Düsseldorf residiert hatten und Worms nicht kurfürstliche Residenz werden wollte, entschloß sich der Kurfürst Karl Philipp 1716 nach unerfreulichen Auseinandersetzungen mit den Heidelbergern, Mannheim zu seiner Residenz zu machen. Er verfügte die Aufgabe der Festung Friedrichsburg und errichtete an ihrer Stelle ein gewaltiges Schloß, das, zusammen mit der Stadt, in einen Bastionenkranz gelegt wurde. Die ansäßig gewordenen protestantischen Flüchtlinge bildeten eine wirtschaftstüchtige Bevölkerung. Das 18. Jahrhundert, das Zeitalter des Merkantilismus mit seiner Idee der staatlichen Planwirtschaft, half mit, die Stadt etwas werden zu lassen.

Hauptförderer war natürlich der Hof. Es entstand die wunderschöne Barockstadt, und gleichzeitig entwickelte sich Mannheim zu einem einzigartigen Kulturzentrum. Neben der Galerie, welche später zum Grundstock der Münchner Pinakothek wurde, finden wir Theater und Oper, eine wissenschaftliche und eine künstlerische Akademie. Stamitz begründete durch seine neuen Ideen die «Mannheimer Schule», die auch Mozart nicht unbeeinflußt ließ. «Alexander von Humboldt entwickelte in Mannheim die Anfänge der Klimatologie, der Hofastronom Mayer entdeckte erstmals die Doppelsterne. Lessing holte sich entscheidende Anregungen, und nicht zufällig floh Schiller mit dem Manuskript seiner «Räuber» gerade hieher. Bevor Weimar sich zur Höhe der literarischen deutschen Klassik erhoben hatte, war Mannheim in viel umfassenderem Sinne Magnet des kulturellen Lebens in Deutschland. Die Mannheimer Akademie der Wissenschaften überstrahlte die Universität Heidelberg bei weitem.» ${ }^{4}$ 


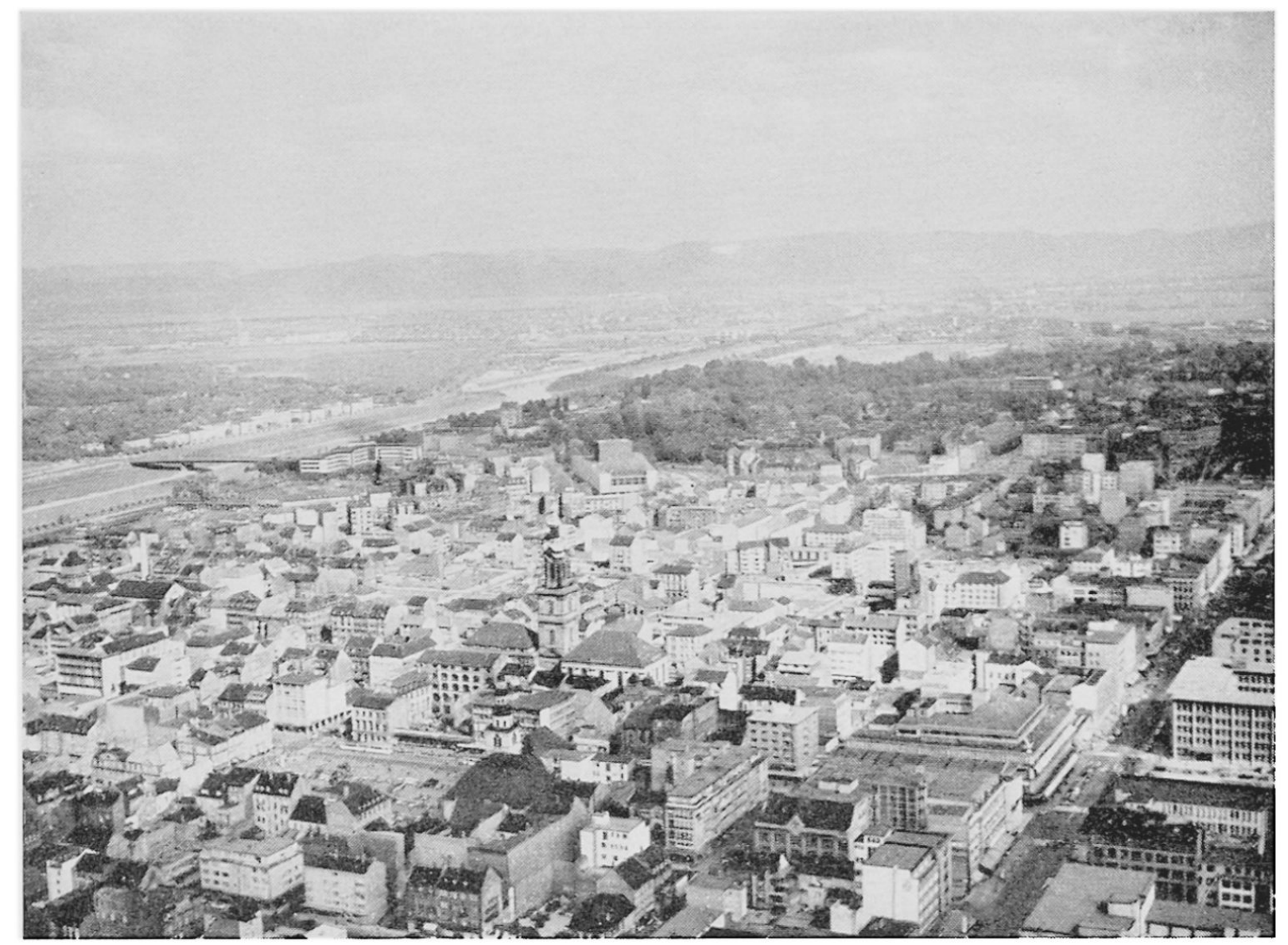

Mannheim. Blick auf östliche Innenstadt, kanalisierten Neckar und Odenwald mit Bergstraße. Photo Bildarchiv des Verkehrsvereins Mannheim.

Diese Blütezeit wurde durch äußere Umstände jäh unterbrochen, und eine Folge von Rückschlägen ließ Mannheim einen sturzartigen Abstieg von der glänzenden Metropole zur bedeutungslosen Stadt durchmachen:

1. 1778 wird der Kurfürst Karl Theodor durch einen Erbgang Bayrischer König und somit gezwungen, seine Residenz nach München zu verlegen. Das bedeutet für Mannheim eine Katastrophe, denn 3000 Personen verlassen die Stadt. Diese, welche bisher als Hauptfunktion die Versorgung des Hofes hatte, sieht sich dadurch der Lebensgrundlage beraubt. Das kulturelle Leben hört bald vollständig auf.

2. Die Revolutionskriege haben französische und österreichische Belagerungen, BeschieBungen und Erstürmungen zur Folge. 1800/1801 schleift man die nutzlos gewordene Festung.

3. Die Kurpfalz wird aufgelöst. Das linksrheinische Gebiet kommt zu Frankreich und wird erst nach Waterloo wieder deutsch. 1816 fällt es teils an Bayern (heutige Pfalz), teils an Hessen (Rheinhessen). Der rechtsrheinische Teil der Kurpfalz gehört seit 1 So3 zum Herzogtum Baden, dessen Residenzstadt Karlsruhe ist. Mannheim liegt jetzt im äußersten NW dieses Territoriums. Das alles hat zur Folge, daß Mannheim zu einer funktionslosen Grenzstadt absinkt, die ihres natürlichen Hinterlandes beraubt ist.

4. 1824 wird in der alten Rheinschanze, jetzt auf Bayrischem Territorium gelegen, ein Hafen angelegt, und es entwickelt sich dort ein kleiner Handels- und Umschlagplatz. Er erhält den Namen Ludwigshafen und tritt als Gegenspieler zu Mannheim auf, das erst seit 3o Jahren einen kleinen Rheinhafen besitzt.

Die Bürger- und Handelsstadt. Die Rückschläge ließen die Mannheimer nicht verzagen, wie eigentlich zu erwarten gewesen wäre. Sie spornten die Bürgerschaft im Gegenteil an.

Gewaltige Energien wurden aufgewendet, mit dem Ziel, Mannheim zur Handelsstadt zu machen. Diesem Streben kam im richtigen Augenblick die Rheinkorrektion zu Hilfe, welche Mannheim zum Endpunkt der Rheingroßschiffahrt werden ließ. Hafen- 
anlagen entstanden, in welchen sich der Umschlag vom Schiff auf die Straße, später auf die Eisenbahn vollzog.

Die führenden Mannheimer Bürger unternahmen alles um ihre Stadt zum «Handels- und Verteilplatz der Güter für ganz Südwestdeutschland, die Schweiz, das Elsaß und das östliche Frankreich ${ }^{5}$ zu machen. Das gelang ihnen auch. Finanz, Handel und Schiffahrt dominierten im Wirtschaftsleben und waren sichtbare Zeichen des steilen Aufstieges. Der systematische Ausbau der Eisenbahnen ließ Mannheim zu einem «verkehrsgeographischen Punkt erster Ordnung» ${ }^{6}$ werden.

$\mathrm{Hand}$ in $\mathrm{Hand}$ mit dem materiellen Wohlstand ging eine durch die geistig rege und aufgeschlossene Bürgerschaft getragene Wiederbelebung der ehemaligen Kulturblüte. Teils durch die Wirren der Revolution von 1848 bedingt, teils aber auch als Folge anderer Ursachen, stellte sich gegen die Mitte des 19. Jahrhunderts auf dem Lande ein Lebensmittelmangel, vor allem eine Getreideknappheit ein. Die Landbevölkerung verzehrte mehr, als produziert wurde. Mannheims Getreidezufuhr stockte. So kam es 1846 zur Errichtung der ersten Getreidetransitlager und einige Jahre später zur Gründung einer Getreidebörse. ${ }^{7}$

In den fünfziger Jahren siedelten sich die ersten Industrien an. Banken entstanden und ersetzten jene von Frankfurt und Basel als Kreditgeberinnen. 1862 durchfloß der Rhein zum ersten Mal den neugeschaffenen Friesenheimer Durchstich nördlich der Stadt. 1867 war die zweigleisige Eisenbahnbrücke über den Rhein betriebsbereit. Im Zuge dieser stürmischen Fortentwicklung blieb ein damals unbedeutend scheinendes Ereignis völlig unbeachtet: 1865 verlegte Friedrich Engelhorn gezwungenermaßen seine Badische Anilin- und Sodafabrik (BASF) von Mannheim nach Ludwigshafen.

Der ständig steigende Hafenumschlag erforderte die mehrmalige Erweiterung der Hafenanlagen. An der Neckarmündung entstand in der zweiten Hälfte des letzten Jahrhunderts der «größte Binnenhandelshafen des europäischen Festlandes, von den Kohlenhäfen des Ruhrgebietes abgesehen.» 8

Doch Rückschläge blieben Mannheim auch jetzt nicht erspart. Und es war auch gut so. Die technischen Mittel erlaubten in den neunziger Jahren die Korrektion der Oberströme: des Rheins bis Basel und des Neckars. Es bestand somit die akute Gefahr, daß Mannheim seine verkehrsgeographische Schlüsselstellung verlieren und der Handel weiter flußaufwärts wandern würde. ${ }^{9}$

Die Industriestadt. Dem konnte die Stadt nach Ansicht der führenden Männer nur so erfolgreich entgegenwirken, daß sie versuchte, «mit allen Mitteln Industrie zu fixieren».10 Man nahm große Eingemeindungen vor und konnte nun auf städtischem Boden ungestört planen und verwirklichen. Der Friesenheimer Altrhein (entstanden durch den Friesenheimer Durchstich im $\mathrm{N}$ der Stadt) wurde zum Industriehafen ausgebaut und mit den notwendigen Bahnanschlüssen versehen. Dort entstand das damals größte Zellstoffwerk der Welt, die heutige Zellstoff Waldhof AG mit 11000 Arbeitern, ferner eine Papierfabrik, die Vereinigten Jutespinnereien, der Verein Deutscher Oelfabriken, Chemische Industrie, Holzindustrie usw. Durch das Abwandern der alten Mühlen vom Odenwaldrand an den Industriehafen und durch die Neugründung von Betrieben in der gleichen Gegend entstand das größte kontinentaleuropäische Mühlenzentrum, welches heute $6 \mathrm{Großbetriebe} \mathrm{umfaßt.} \mathrm{(Die} \mathrm{Walzmühle}$ in Ludwigshafen hat eine Tageskapazität von 750 t.) Ganz Südwestdeutschland und teilweise auch die Schweiz wurden von ihm beeinflußt.11 Noch heute ist der Anteil von Getreide und Mahlprodukten am Hafenumschlag ansehnlich: ${ }^{12}$

Gesamtumschlag in den Mannheimer Häfen $1958 \quad 6637100 \mathrm{t}=100 \%$
1958 Ankunft: Getreide, Mais . . . . . . . $590400 \mathrm{t}$
1958 Abfahrt: Getreide, Mais, Mehl, Futtermittel . $\quad \frac{298400 \mathrm{t}}{888800 \mathrm{t}}=13,3 \%$


Das beim Industriehafen errichtete, im Schnittpunkt der Saar- und Ruhrferngasleitungen gelegene Gaswerk besitzt eine Kapazität von 470000 m³$^{3}$ Tag und kann zur

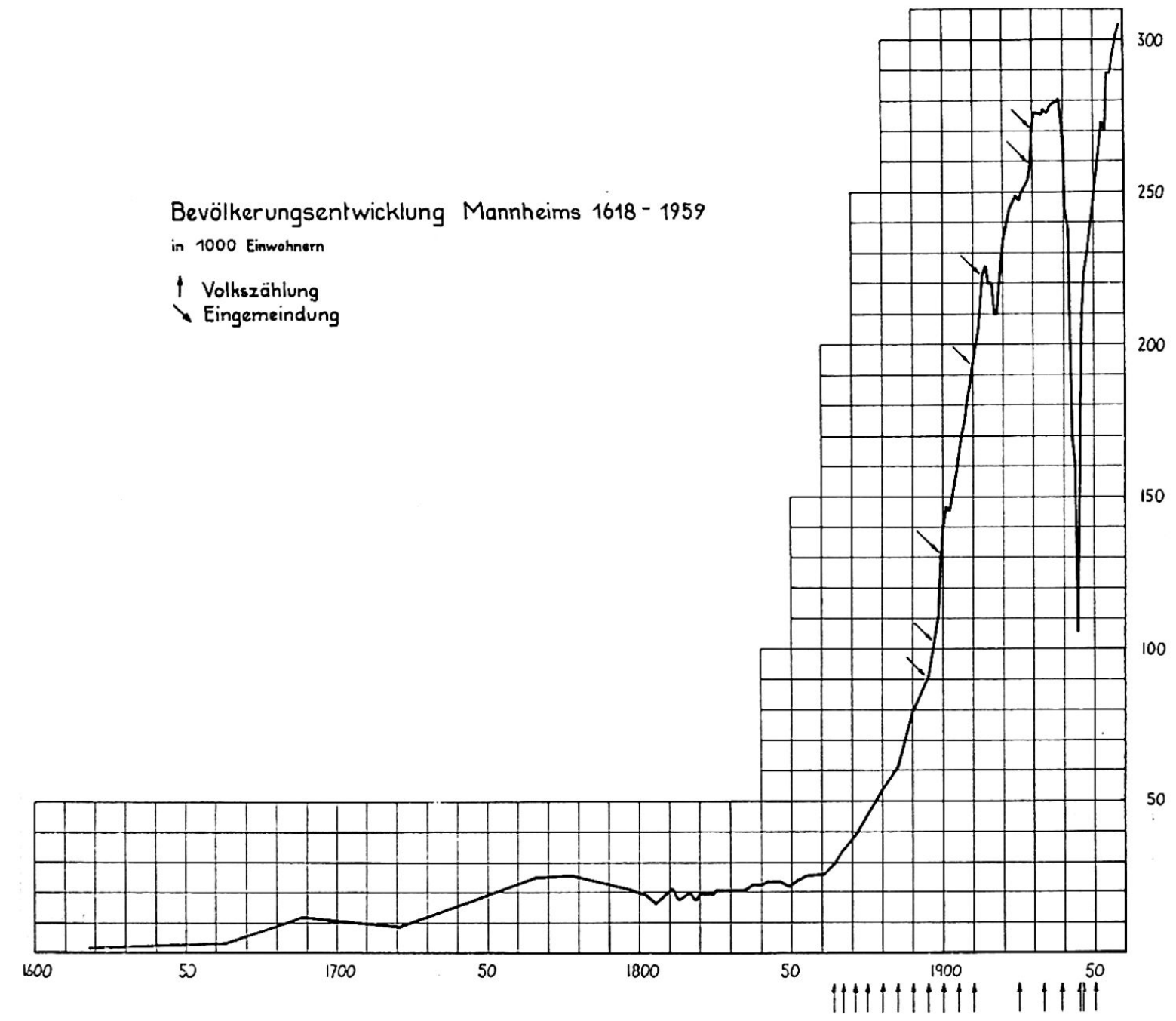

Fig. 1

Speisung des Ferngasleitungsnetzes auf $600000 \mathrm{~m}^{3} / \mathrm{Tag}$ ausgebaut werden. ${ }^{13}$. (Zum Vergleich: Das Gaswerk Zürich in Schlieren lieferte 1958 im Durchschnitt ca. $190000 \mathrm{~m}^{3}$ /Tag.) 14

Im Südteil des Stadtgebietes, bei Rheinau, wurde ein zweiter Industriehafen angelegt, was ebenfalls erst die Eingemeindungen ermöglichten. Dort befinden sich heute das Großkraftwerk, ein Stahlwerk, die Süddeutschen Kabelwerke, eine Sunlight Fabrik und viele andere Betriebe. Der Kohlenhandel und die Brikettfabrikation siedelten sich ausschließlich beim Rheinauhafen an, sodaß dieser in erster Linie zum Kohlenverteiler für Südwestdeutschland wurde. ${ }^{15}$

Die zu verschiedenen Malen zwischen 1895 und 1930 vorgenommenen Eingemeindungen ließen zwar die Gemarkungen der Stadt unglaublich anschwellen (von 18942384 ha auf 193014368 ha, heute 14495 ha, Zürich 19579189 ha). Der dadurch entstandene Nachteil großer Entfernungen (N-S Erstreckung fast $20 \mathrm{~km}$ ) wird aber bei weitem durch folgende Vorteile aufgewogen:

a) Eine Dezentralisation der Wohngebiete und das Dazwischenschalten großer Grünzonen, die heute noch landwirtschaftlich genutzt werden, wird sehr erleichtert.

b) Jegliche Planung erhält durch die Gemarkungsausmaße neue Möglichkeiten und unterliegt weniger räumlichen Beschränkungen. 


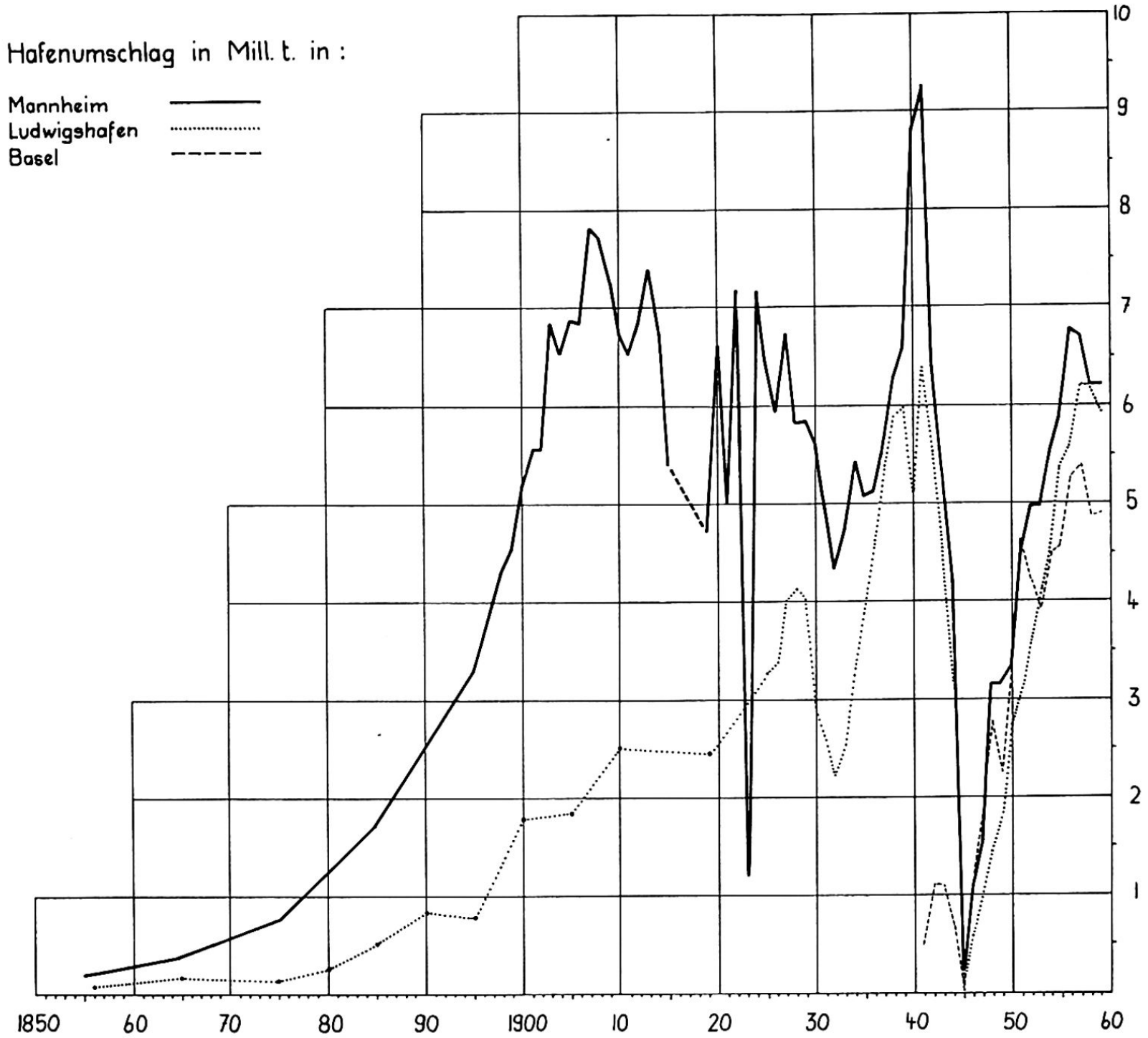

Fig. 2

c) Industriegebiete können im $\mathrm{N}$ und $\mathrm{S}$ der Altgemarkung entstehen, was im Bezug auf die Abgase (Westwinde) unbedingt erwünscht ist.

Nach und nach haben sich in Mannheim gegen 300 (1958 279) ${ }^{16}$ Fabriken aller Größe und Art niedergelassen, darunter Großbetriebe wie Daimler Benz (hier schuf Karl Benz 1885 sein erstes Auto), Heinrich Lanz (eine Fabrik landwirtschaftlicher Maschinen wie Bucher Guyer), Brown Boveri (ein Tochterwerk der BBC in Baden, das heute größer ist als die Mutterfabrik) usw.

Ein Blick auf die Bevölkerungsentwicklung und den Entwicklungsgang des Hafenumschlages zeigt den beinahe amerikanischen Aufstieg Mannheims.

Die Entwicklung von Ludwigshafen

- Wie erwähnt, entstand im zweiten Viertel des 19. Jahrhunderts in der Rheinschanze der Hafen- und Handelsplatz Ludwigshafen. 1852 löste man aus zwei Gcmeinden Landareale heraus, und Ludwigshafen wurde zur politischen Gemeinde. Als Umschlageplatz für die bayrische Pfalz hatte die Siedlung eine Stellung erlangt, die Bayern bewog, sie 1859 zur Stadt zu erheben. Dies geschah aber vor allem auch mit der Absicht, Ludwigshafen am Aufstieg Mannheims teilhaben zu lassen. Man baute neue Lager- und Handelshäuser. Daß die BASF durch Verschulden Mannheims nach Ludwigshafen übersiedeln mußte, bedeutete für die Stadt eine große wirtschaftliche Chance. Die unglaubliche Entwicklungsdynamik dieser Fabrik (1959 gegen 45000 
Beschäftigte) zog weitere chemische Unternehmen an, und das gab Ludwigshafen eine völlig neue Funktion. Es wurde von der ausschließlichen Handelsstadt zur ebenso einseitig orientierten Industriestadt: zur deutschen Chemiestadt. Die chemische Industrie, im besonderen die BASF, zeigte eine ungeheure Expansionskraft und ließ die Stadt rasch groß werden. Im Bezug auf die Geschwindigkeit der Bevölkerungsentwicklung (Fig. 3) und das Ansteigen des Hafenumschlages stand Ludwigshafen Mannheim nicht nach (Fig. 2).

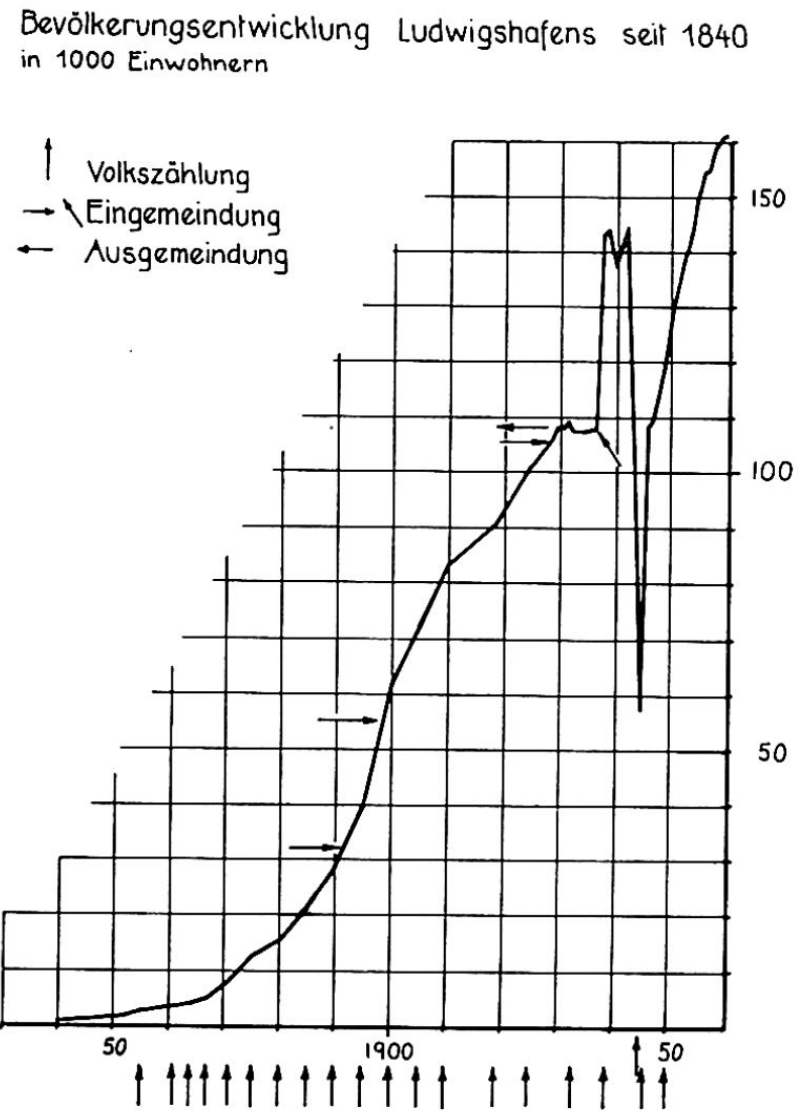

Fig. 3

Leider ließ dieses Vorwärtsstürmen der Stadt kaum Zeit, sich un ein städtebaulich befriedigendes Wachstum zu kümmern. Erst im Wiederaufbau nach dem zweiten Weltkrieg konnte vieles geändert und verbessert werden.

\section{DiE HEUTIGEN VERH ÄLTNISSE DER BEIDEN STÄDTE}

Nach dem zweiten Weltkrieg erholten sich Mannheim und Ludwigshafen nur langsam von dessen katastrophalen Folgen. Bevölkerungs- und Hafenumschlagsentwicklung zeigen das eindrücklich (Fig. 1,2 und 3). Erst in den frühen fünfziger Jahren erreichten die beiden Städte den Vorkriegsstand der Bevölkerung wieder. Mannheim mit seinen über 300000 Einwohnern (Dezember 1959305712 ) und Ludwigshafen mit über 160000 Einwohnern (November 1959 161 270), sind Großstädte geworden. Darin zeigt sich eine weithin sichtbare Folgeerscheinung der im Vorangehenden geschilderten Entwicklung. In ihrer Art unterscheiden sich beide Städte sehr voneinander.

Mannheim, die Stadt der vielfältigen Industrie, das Handels-, Finanz- und Hafenzentrum mit der Produktenbörse, dem Nationaltheater und der Wirtschaftshochschule (ähnlich der Handelshochschule St. Gallen mit 19591400 Studenten), fühlt sich als die stärkere von beiden. Sie ist sich ihrer Aufgabe als Erbin geistig-kultureller 
Traditionen der Vergangenheit bewußt und fördert Kultur und Wissenschaft in hohem Maße.

Ludwigshafen, die Stadt der Chemie, der Monoindustrie, die kleinere, fühlt sich leicht benachteiligt gegenüber ihrer größeren Nachbarin. Es sind denn auch Spannungen zwischen den beiden Städten spürbar, die sich bei der Lösung gemeinsamer Aufgaben unerfreulich auswirken. Daran tragen aber nicht die beiden Städte allein Schuld. Vielmehr liegen die Ursachen vieler Unstimmigkeiten in der unglücklichen Grenzziehung am Rhein. Ein weiterer Grund bildet das Eisenbahnproblem (Kapitel III).

Der einheitliche Wirtschaftsraum Mannheim-Ludwigshafen gehört drei Ländern an: Baden-Württemberg, Rheinland-Pfalz und selbst noch etwas Hessen (Fig. 5). Mannheim, im nördlichsten Baden und Ludwigshafen im E der Pfalz, befinden sich noch fast in der gleichen Grenzlage, wie sie durch den Wiener Kongreß geschaffen wurde. Das alte politische Gebilde der Kurpfalz entsprach weitgehend den natürlichen Gegebenheiten der Oberrheinischen Tiefebene. Mit einem derart bedeutungsvollen wirtschaftlichen Kernraum im Zentrum, wäre die Kurpfalz heute ein absolut lebensfähiger Organismus (ähnlich wie z. B. Hessen mit dem zentralen Rhein-Main-Raum). Doch alle Anstrengungen zur Neugliederung des Bundesgebietes nach dem zweiten Weltkrieg verliefen trotz der starken Unterstützung durch Historiker und Geographen, welche mit überzeugenden Argumenten aufwarteten, im Sande. Statt einer Einheit, besteht die paradoxe Situation, da $\beta$ in dem geschlossenen Wirtschaftsraum zwei Hafenverwaltungen, zwei Arbeitsämter und drei Eisenbahndirektionen die politische Zerschneidung des Raumes eher noch verstärken. Mannheim hat nicht einmal ein Postcheckamt; dieses befindet sich in Karlsruhe. Mainz, Wiesbaden und Stuttgart sprechen als Landeshauptstädte aus weiter Entfernung die entscheidenden Worte.

Die beiden Städte, welche zusammen 500000 Menschen zählen und den stärksten Wirtschaftsplatz im Oberrheingebiet bilden, besitzen kaum Bedeutung als Mittelpunkte staatlicher Verwaltung. Sie sind weder Sitz höherer Landes- noch Bundesbehörden. Bezeichnend ist, daß auch eine derart ausgeprägte politisch-verwaltungsmäßige Benachteiligung nicht verhinderte, da $\beta$ sich beide Städte ein natürliches Einzugsgebiet schaffen und dieses sogar ständig ausbauen konnten, ohne sich um Grenzen zu kümmern. Das sei an Hand der Pendelwanderungen noch anschaulicher gezeigt.

Fig. 4 stellt die Herkunftsorte der Einpendler nach Mannheim dar. ${ }^{20}$ Die Größe der Zeichen entspricht deren Anzahl. Hieraus ergibt sich ein gutes Bild von der derzeitigen (1958) Ausdehnung des Mannheimer Wirtschaftsraumes. Die ganze Oberrheinische Tiefebene zwischen Speyer und Worms, Teile des Pfälzer Waldes, des Odenwaldes und weite Gebiete des Kraichgaus liegen in dessen Einflußbereich. Die recht zufälligen und zahlenmäßig nicht ins Gewicht fallenden Herkunftsorte außerhalb dieses Raumes, wie Kaub am Rhein, Wiesbaden, Frankfurt, Offenbach und Darmstadt, sowie Karlsruhe und Kaiserslautern, dürfen für seine Abgrenzung ruhig vernachläßigt werden. Schon bei dieser Art der Darstellung kann man gewisse Schwerpunktsbildungen erkennen. Gebiete, die sehr viele Pendler nach Mannheim entsenden, heben sich von Gegenden ab, für welche eine Verdienstmöglichkeit in der Stadt eine geringere Rolle spielt, oder die ev. schon im Einflußbereich einer anderen Großstadt liegen, wie z. B. Frankfurt, Stuttgart oder Karlsruhe. So stellen die Siedlungen der Ebene den Hauptharst aller täglichen Zuzüger, während die Gebirgsgegenden und der stark bäuerliche Kraichgau in dieser Beziehung geringere Bedeutung haben. Es fällt vor allem auf und muß besonders unterstrichen werden, da $\beta$ große Pendlermassen selbst aus den benachbarten Bundesländern Rheinland-Pfalz und Hessen nach Mannheim fahren. So kamen 1958 aus Nordbaden 33000 Pendler, aus Hessen 15000 u. aus der Rheinpfalz deren 9000 dorthin, total also 57000 Pendler. Da aber in der verwendeten Statistik nur diejenigen Einpendler erfaßt wurden, die aus Gemeinden kamen, welche 1958 mehr als 20 Pendler nach Mannheim stellten, ist für die Stadt mit täg- 


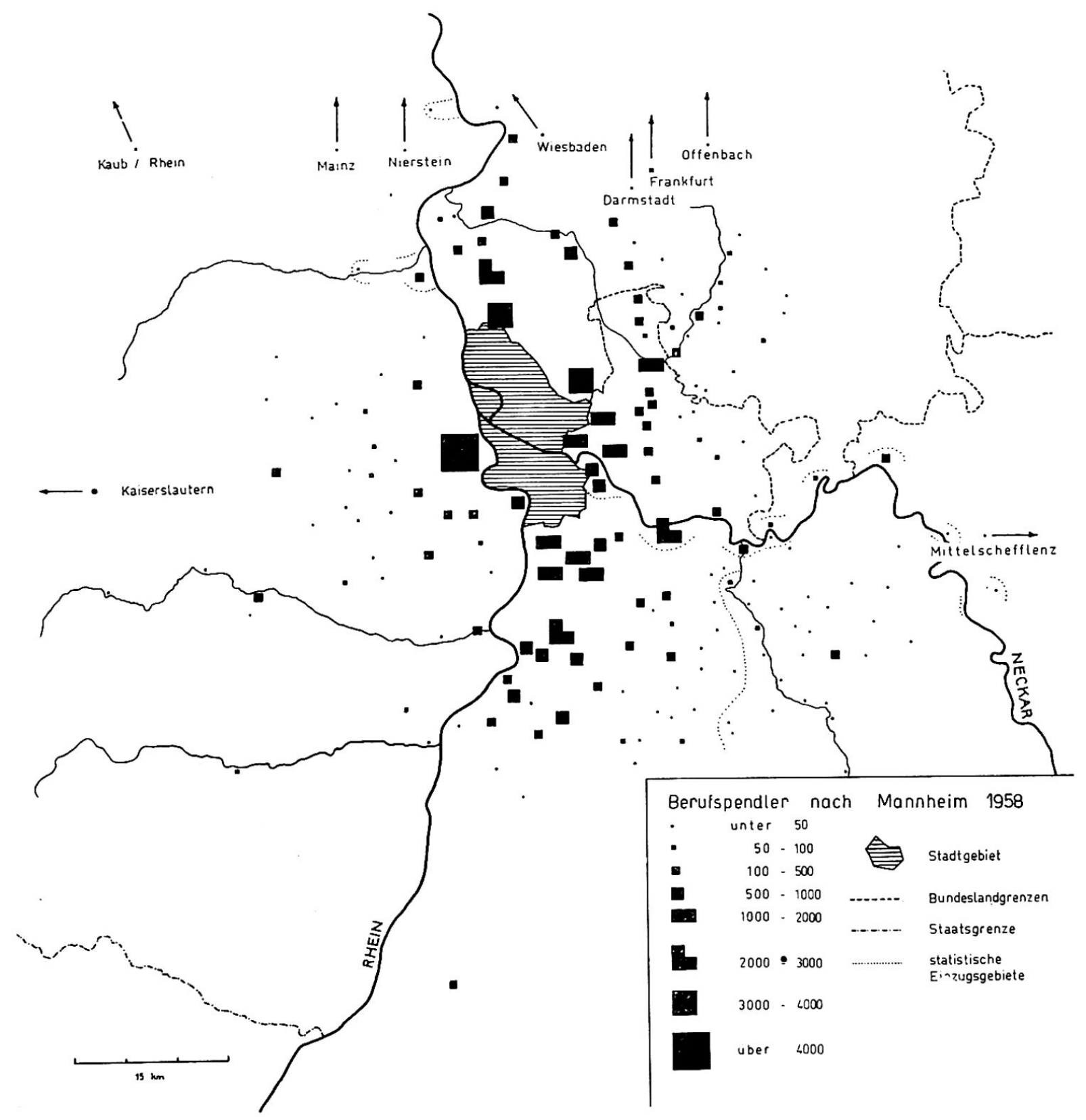

Fig. 4

lich 60000 Berufspendlern zu rechnen. (Zürich 195541 000.) Diese Zahlen lassen die überragende Bedeutung Mannheims als Arbeitsplatz erkennen. (In der gesamten Industrie waren 195865769 Arbeiter und 19146 Angestellte, also 84915 Menschen beschäftigt.) ${ }^{21}$

Das Bild wird noch klarer, wenn der absoluten Darstellung eine relative gegenübergestellt wird, welche die prozentualen Anteile der täglich in Mannheim arbeitenden Personen an der Gesamteinwohnerzahl ihrer Wohngemeinde zeigt (Fig. 5). Ludwigshafen, das fast 6000 Pendler stellt, verschwindet dabei. Auch das Odenwaldgebiet und die Kraichgaulücke werden jetzt signaturenlos. Das heißt: Mannheim ist der Arbeitsplatz für den rechtsrheinischen, also den südhessischen und den nordbadischen Teil der Rheinebene. Im Umkreis von $30 \mathrm{~km}$ um Mannheim lebten Ende 1957 in den Gemeinden der oben erwähnten Pendlerstatistik inkl. Mannheim selbst über 1,5 Mio Menschen. Würden alle Siedlungen berücksichtigt, wären es noch wesentlich mehr. 1958 gab es nur 5400 Auspendler, vor allem wohl nach Ludwigshafen in die BASF.22

Links des Rheines hat Ludwigshafen die gleiche Bedeutung für die Pfalz. Die bei- 


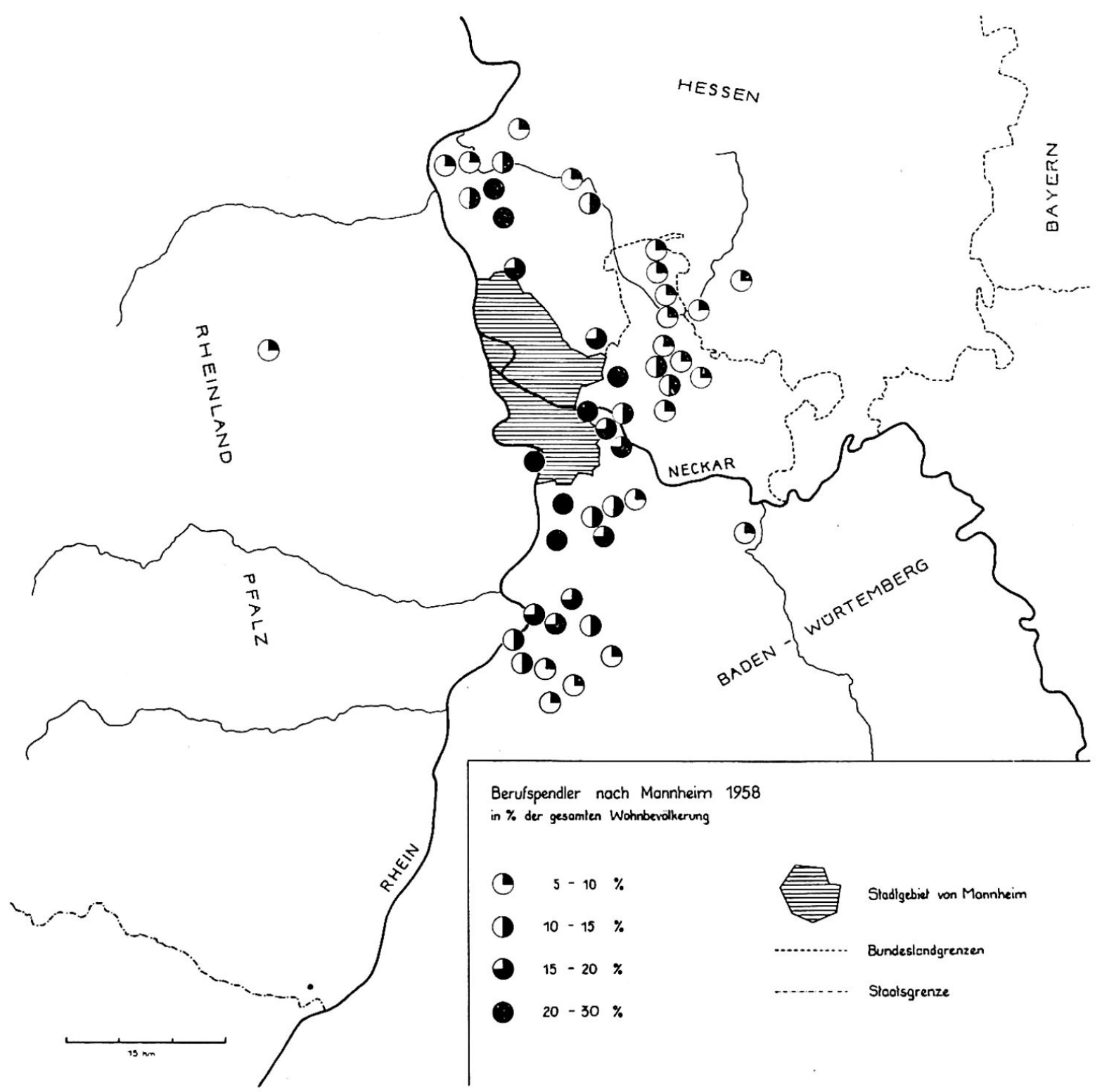

Fig. 5

den Städte konkurrenzieren einander somit praktisch nicht, vielmehr ergänzen sie sich gegenseitig, wirken gemeinsam als ein großer Magnet und ziehen täglich gegen 100000 Pendler in ihre Gemarkungen. Diese geringe Konkurrenz mit Bezug auf die Pendler gilt z. B. nicht hinsichtlich der Stellung Mannheims und Ludwigshafens als Einkaufszentren. Obwohl sich bei beiden eine Citybildung vollzieht und demnach große Teile der Innenstädte reinen Geschäftscharakter angenommen und sich entvölkert haben, hat doch Mannheim in dieser Hinsicht weit größere Bedeutung. Sie ist so groß, daß selbst aus der benachbarten Großstadt Heidelberg Kauflustige zuströmen. Das alles ist den betroffenen Kreisen in Ludwigshafen keineswegs gleichgültig, denn sie wissen, daß viele Leute, auch aus ihrer Stadt und der Pfalz, in Mannheim Einkäufe tätigen. Ein Grund mehr zu gegenseitigen Spannungen. So war denn die Verwaltung der Stadt schon mehrmals nahe dem Entschluß, das einzig mit Mannheim Gemeinsame, nämlich die Straßenbahn, auch noch zu trennen, d. h. die Verbindung über die Rheinbrücke zu erschweren. Glücklicherweise kam es bisher nie so weit.

Die Häfen der beiden Städte, schon getrennt von großer Bedeutung, was auch ein Vergleich mit dem Basler Hafenumschlag und die Aufstellung der größten deutschen Binnenhäfen zeigen, wären als gemeinsamer Hafen der vereinigten Doppelstadt Mannheim-Ludwigshafen umschlagmäßig der zweitgrößte Deutschlands. 
Umschlagsleistungen der Deutschen Binnenhäfcn 195723

\begin{tabular}{|c|c|c|c|c|}
\hline 1. Duisburg-Ruhrort & 16199813 & & 9. Berlin & $1875 \circ 22$ \\
\hline 2. Mannheim & 7211232 & & 1o. Mainz & 1557574 \\
\hline 3. Ludwigshafen & 6213607 & $13+2+839$ & 11. Gelsenkirchen & 1083328 \\
\hline 4. Bremen (Binnenhafen) & 4635938 & & 12. Kehl & $105+910$ \\
\hline 5. Köln & 3550018 & & 13. Worms & 522541 \\
\hline 6. Frankfurt & 3398596 & & 14. Koblenz & $44654+$ \\
\hline 7. Karlsruhe & $2855+15$ & & 15. Offenbach a. Main & 289384 \\
\hline 8. Düsseldorf & 2418949 & & & \\
\hline
\end{tabular}

Mannheim hatte 1957 30935 registrierte Schiffsbewegungen zu verzeichnen 24, Basel im gleichen Jahr 1o 189.25 Von den in Mannheim 1958 registrierten 29 920 Schiffen, waren 18450 deutscher, 8269 niederländischer, 1566 schweizerischer, 935 belgischer, 66o französischer, 36 österreichischer und 4 luxemburgischer Herkunft.26

Das von Kriegsschäden nicht betroffene Heidelberg und das schwer mitgenommene Karlsruhe haben nach dem Kriege die Stagnation der Entwicklung sehr rasch überwunden. Heidelberg zählt heute anderthalb mal so viele Einwohner wie 1939 (1939 84300,1958 125000). Dabei halfen das US-Hauptquartier, die Universität, der Fremdenverkehr und die erstmals zugezogene Industrie entscheidend mit. Auch Karlsruhe hat sich seither sehr stark entwickelt (1939 184500 Einwohner, 1957227 200), wurde eine große Industriestadt, Atomforschungszentrum, Sitz des Bundesgerichtshofes und blieb als Hauptstadt des Regierungsbezirkes Nordbaden Behördenmittelpunkt. So sind zwar Heidelberg und Karlsruhe bevölkerungsmäßig rascher gewachsen als Mannheim und Ludwigshafen. Diese behaupteten jedoch ihre Stellung als Wirtschaftszentren.

\section{HEUTIGE VERKEHRSPROBLEME DES RAUMES}

Eisenbahnfragen. Das Eisenbahnnetz der nördlichen Oberrheinischen 'Tiefebene zeichnet sich durch folgende zwei Besonderheiten aus:

a) Der NS-Verkehr wickelt sich nördlich der Städte Heidelberg, Mannheim und Ludwigshafen auf drei (zwei rechtsrheinischen und einer linkrheinischen) Schnellzugslinien und südlich der genannten Städte bis Karlsruhe auf zwei rechtsrheinischen parallel verlaufenden Eisenbahnlinien ab.

b) Der Rhein-Neckar-Raum weist heute zwei Kopfbahnhöfe (Mannheim und Ludwigshafen) auf, und bis 1955 war auch der Heidelberger Hauptbahnhof als Kopfbahnhof ausgebaut.

Wie es im Laufe der Entwicklung des Schienennetzes, bedingt durch die schon beschriebenen territorialpolitischen Verhältnisse zu diesem heutigen Zustand kam, soll hier weiter nicht untersucht werden. Vielmehr möchte ich dic obigen Tatsachen noch näher erläutern.

Die Schnellzüge von Basel Richtung Norddeutschland und Rheinland benützen bis Karlsruhe die gleiche, rechtsrheinische Linie. Dort teilt sich der Verkehr nach Norddeutschland:

Ein Teil der Züge benützt die direkte Linie über Schwetzingen, Mannheim-Friedrichsfeld nach Frankfurt (Rheintalbahn und Main-Neckar-Bahn).

Der andere Teil fährt via Bruchsal - Heidelberg und mündet in MannheimFriedrichsfeld wieder in die Rheintalbahn.

Die Züge ins Rheinland benützen die Rheintalbahn bis Mannheim und können von hier aus eine links- und eine rechtsrheinische Strecke befahren. Sie gelangen entweder via Rheinbrücke - Ludwigshafen - Worms - Mainz oder über die Riedbahn und Mainz weiter nordwärts. Das gleiche gilt bedingt auch für den Personen-, Eil- und Güterzugsverkehr.

Mannheim wird also vom Norddeutschland - und Heidelberg vom Rheinlandverkehr größtenteils nicht berührt. Das bringt den beiden Städten selbstverständlich nur Nachteile. Die Notwendigkeit der Benützung von Zubringerzügen und das Umsteigen 
verursachen große Zeitverluste für die Reisenden. Der durchgehende Längsverkehr im Rheintal sollte unbedingt Mannheim und Heidelberg tangieren.

Der Mannheimer Hauptbahnhof ist für die Züge, welche auf der Riedbahn verkehren, Kopfbahnhof, für alle übrigen natürlich Durchgangsbahnhof. Um die Spitzkehre auszuschalten, ist geplant, die genannte Linie von Mannheim-Waldhof durch das Hafengebiet direkt in den Hauptbahnhof zu führen. Im Zuge dieses Ausbaues wäre es zugleich möglich, eine Ringbahn um die Stadt zu ziehen, was vor allem den Arbeiterpendlern erlauben würde, direkt ins Industriegebiet zu fahren, ohne noch Straßenbahn und Omnibusse benützen zu müssen. Dringendere Aufgaben verhindern vorläufig die Ausführung dieses Projektes, das eine wesentliche Verbesserung der Verkehrsverhältnisse bringen würde und eines Tages gewiß Wirklichkeit werden wird. Im Zusammenhang mit der Anlage der neuen Straßenzufahrten zur Rheinbrücke, ist der Bahnkörper für die geplante direkte Einführung der Riedbahn schon erstellt worden.

Der Hauptbahnhof Ludwigshafen hingegen ist ein volkommener Kopfbahnhof. Er stellt aus folgenden Gründen das Hauptproblem beim Ausbau des Eisenbahnnetzes dar:

a) Es müssen nicht nur die Züge von und nach dem Rheinland hier einen Lokomotivwechsel vornehmen, sondern auch diejenigen der WE-Linie Saargebiet - Würzburg oder Stuttgart. (Seit der Rückgliederung des Saarlandes hat diese Verbindung stark an Bedeutung gewonnen!) Erhebliche Zeitverluste sind die Folgen und diese wiegen in unserer Zeit schwer. Geht es doch heute vor allem darum, den Eisenbahnverkehr gegenüber dem Straßenverkehr durch Beschleunigung konkurrenzfähig zu halten.

b) Der Bahnhof trennt die Stadt in zwei Teile, erschwert deren einheitliche Entwicklung, aber auch die flüssige Abwicklung des Verkehrs sehr und verunmöglicht einen unbehinderten Zugang zum großen chemischen Werk der BASF.

Aus diesen Gründen ging das Bestreben der Deutschen Bundesbahnen, aber auch der Stadtplaner dahin, beim Wiederaufbau und Ausbau des zerstörten Eisenbahnnetzes den Hauptbahnhof in den W oder S der Stadt zu verlegen, damit er Durchgangsbahnhof würde. Dieser Plan scheiterte am Widerstand der Stadt Ludwigshafen. Gründe dafür waren die enorm hohen Baukosten, die Notwendigkeit den Hafen und die BASF trotzdem durch das heutige Bahnhofsgebiet mit dem neuen Bahngelände verbinden zu müssen und letztlich auch die geheimen Befürchtungen, bei der Projektverwirklichung von den Schnellzügen ohne Halt durchfahren zu werden. Man glaubte die Schnellzugshalte durch die Beibehaltung des Kopfbahnhofes erzwingen zu können. Wollte die Bundesbahn ihre Züge beschleunigen, so mußte sie trotz allem eine Lösung suchen. Es wurde für die linksufrige Rheinlandlinie, ähnlich wie in Olten für die Gotthardgüterzüge, ein Verbindungsgeleise gebaut, um die Spitzkehre zu vermeiden. Auch die Züge von und ins Saargebiet berühren den Hauptbahnhof Ludwigshafen immer weniger. Somit wird dieser vom Durchgangsverkehr notwendigerweise abseits gelassen. In Ludwigshafen werden je länger desto weniger Schnellzüge Halt machen. Folgende Angaben mögen das veranschaulichen: Im Sommer 1959 verkehrten im Mannheimer Hauptbahnhof täglich 101 Schnell- und 106 Personenzüge, total also 207 Züge. Im Hauptbahnhof Ludwigshafen hingegen waren es nur 59 Schnell- und 84 Personenzüge, total 143 Züge. Die Einsicht, daß eine Bahnhofsverlegung trotz der Kosten nicht nur verkehrspolitisch, sondern auch städtebaulich absolut von Vorteil wäre, hat sich leider zu spät durchgesetzt. Der Zeitpunkt ist engültig verpaßt. Es ist nicht einzusehen, weshalb die beiden Städte, die doch eine Einheit bilden, in nur zwei km Luftdistanz zwei Hauptbahnhöfe haben müssen. Eine Fahrt mit der Straßenbahn zum Mannheimer Hauptbahnhof hinüber kann nicht nur weniger lang dauern, als zu demjenigen von Ludwigshafen, sondern Mannheim bietet auch die besseren Verbindungen. Die Tendenz, als Ludwigshafener in Mannheim die Züge zu besteigen, wird sich mit der Verbesserung der Rheinübergänge nur noch verstärken.

Heute sind alle NS-Linien des Rheintales elektrifiziert, also auch die Geleisean- 
lagen der beiden Städte. Die Zufahrtsrampe zur zweigeleisigen Eisenbahnbrücke von der Mannheimer Seite her ist sehr steil - wegen des Rheinverkehrs mußten die Brückenjoche sehr hoch gelegt werden - so daß beim früheren Dampfbetrieb eine $\mathrm{Zu}$ satzlokomotive die Züge von hinten stoßen mußte. Dieses umständliche und zeitraubende Manöver fällt heute, der stärkeren Lokomotiven wegen, glücklicherweise weg.

Mannheim hat neben Hagen im Ruhrgebiet den größten und am stärksten frequentierten Verschiebebahnhof Deutschlands. Er liegt im SE des Stsdtgebietes zwischen dem Hauptbahnhof und der Station Mannheim-Friedrichsfeld. In ihm werden täglich über 6000 Wagenverschiebungen vorgenommen. ${ }^{27}$ Seine Bedeutung für ganz Südwestdeutschland steigt noch mit der wirtschaftlichen Einverleibung des Saarlandes ins westdeutsche Wirtschaftsgebiet.

Straßenverkehrsfragen. Mit dem ständig fortschreitenden Ausbau des bundesdeutschen Autobahnnetzes, rücken die beiden Städte immer mehr in dessen Zentrum. In Bälde werden die Strecken nach Saarbrücken und südwärts bis Basel betriebsbereit sein. Dann ist für den Abschnitt Heidelberg - Mannheim, der schon 1957 von täglich 45000 Autos befahren wurde, eine nochmalige Frequenzzunahme zu erwarten. Eine Entlastung kann nur durch den Ausbau des Bundesstraßennetzes (bei uns wären das die Straßen 1. Klasse) des Rhein-Neckar-Raumes erzielt werden. Die Verbreiterung der Autobahn fällt außer Betracht.

Für die Bundesstraßen gibt es zwischen Speyer und Worms, also auf $40 \mathrm{~km}$ Flußlänge, nur die eine Brücke zwischen Mannheim und Ludwigshafen, die sog. Stadtbrücke. Und gerade diese Tatsache stellt die Straßenbaubehörden vor die größten Probleme. Drei Bundesstraßen (die B 37, B 38 und B 44) sammeln die Verkehrsströme der Pfalz Richtung $\mathrm{E}$ und führen diese zur Rheinbrücke zwischen Ludwigshafen und Mannheim. Auf der Mannheimer Seite verteilen deren Fortsetzungen (B 36, B 37, B 38 und B 44) den Verkehrsstrom wieder. Es bestehen Anschlüsse an die Autobahn nach allen Richtungen. In der entgegengesetzten Richtung spielt sich derselbe Vorgang $a b$.

Neben diesem ausgesprochenen WE und EW Durchgangsverkehr, der natürlich die städtischen Straßen sehr belastet, dient die Rheinbrücke auch dem zwischenstädtischen Verkehr. Die Brückenpfeiler tragen außerdem noch zwei Straßenbahn- und die beiden Bundesbahngeleise. Aus all diesen Gegebenheiten resultieren ganz gewaltige Verkehrsfrequenzen. So benützen täglich 32-34000 Automobile die Straßenbrücke (ohne Fußgänger, Velofahrer und Trams) und 1970 sollen es deren 60000 sein. Zum Vergleich sei gesagt, daß die Quaibrücke in Zürich 1957 von nur 19000 Autos benützt wurde. Hieraus wird klar, daß sich die Straßenverkehrsfragen ganz einfach als Rheinbrückenproblem umschreiben lassen.

Die Planungsbehörden entschlossen sich nach genauen Untersuchungen zuerst einmal die Stadtbrücke samt ihrer Zufahrten so auszubauen, daß sie den anfallenden Verkehr bewältigen können. Erst nach dieser Entschlußfassung trat man an die Frage. einer zweiten Straßenbrücke heran. Man muß dabei zwischen einer Nordbrücke beim heutigen Hauptbahnhof Ludwigshafen oder einer Südbrücke wählen oder sich für beide entscheiden. Die Untersuchungs- und Planungsarbeiten sind heute in vollem Gange.

Der Ausbau des bestehenden Rheinüberganges warf große Probleme auf und verlangte Entscheidungen, die sich finanziell schwer auswirken werden.

Der Rhein beschreibt oberhalb und unterhalb der Stadtbrücke eine leichte Links. biegung. Diese verunmöglichte eine weitere Verlängerung der Brückenpfeiler, denn die Schiffe wären dann, laut Erklärung der Rheinschiffahrtsdirektion, aus Strömungsgründen gewissen Gefahren ausgesetzt. Deshalb konnte die Straßenbrücke nur wenig verbreitert werden (man legte das Trottoir frei ausladend an und gewann so etwas Fahrbahnbreite), und auch die Bundesbahnen mußten darauf verzichten, ein drittes 
oder sogar viertes Geleise zu legen. Um die Leistungsfähigkeit der Stadtbrücke zu steigern, war man gezwungen, einen entsprechenden Ausbau der beiderseitigen $\mathrm{Zu}$ - und Abfahrtswege vorzunehmen. Deren Neuanlage sollte in Zukunft dafür Gewähr bieten, daß keine Stauungen mehr entstehen würden.

Auf der Ludwigshafener Seite fand man die Lösung darin, daß Teilströme des Durchgangsverkehrs auf einer Hochstraße um die Innenstadt herumgeführt werden und der Stadtverkehr kreuzungsfrei zu- und abgeleitet wird. Auf der Mannheimer Seite baute man ein kreuzungsfreies, sehr kompliziertes Rampensystem (getrennte Wege für Autos, Straßenbahn, Radfahrer und Fußgänger) auf dem Gelände des ehemaligen Schloßparkes, das Zuleitung und Verteilung des Verkehrs reibungslos bewältigen kann. Hand in Hand damit ging und geht heute noch eine durchgreifende Sanierung der innerstädtischen Verkehrsverhältnisse in den beiden Städten. Man führt vor allem konsequent den Einbahnverkehr ein. Mannheim im besonderen muß auch seinen Bahnhofplatz umgestalten, die Zufahrten zum Stadteil Lindenhof (zwischen Rhein und Hauptbahnhof gelegen) verbessern und mit sehr durchdachten Verkehrsführungen die Sperrwirkung des mächtigen Schloßbaues aufzuheben suchen.

Die neue Stadtbrücke und die beiderseitigen Zufahrten, mit ungeheurem Kostenaufwand erbaut, sind seit November letzten Jahres vollständig in Betrieb und bewähren sich bestens.

\section{A N MERKU N GEN}

1 Lit. 13 S. 6 - 2 Lit. 25 - 3 Lit. 13 S. 7 - 4 Lit. 13 S. 13 - 5 Lit. 13 S. 20 - 6 Lit. 13 S. 20 7 Lit. 13 S. 23 - 8 Lit. 13 S. 28 - 9 Lit. 13 S. 28 - 10 Lit. 13 S. 28 - 11 Lit. 13 S. 29 und Lit. 12 S. 194/195 - 12 Lit. 22 Stat. Jahresbericht 1958 - 13 Lit. 6 S. 152/153 - 14 Lit. 23 S. 312 - 15 Lit. 13 S. 29 - 16 Lit. 20 Stat. Jahresbericht 1958 S. 42 - 17 Angaben 1618 - 1953 aus Lit. 6 S. 22, ab 1954 aus Lit. 20 S. 2 - 18 Mannheim: Lit. 6 S. 122 mit Angaben bis 1953, ab 1954 Zahlen aus Lit. 22. Ludwigshafen: Für die Zeit 1856 - 1925 liegen nur vereinzelte Angaben vor, und die Umschlagskurve zeigt demnach ein sehr ungenaues Bild. Die größeren Punkte bedeuten erhalten gebliebene Angaben vor 1925 aus Lit. 15 S. 66. Zahlen ab 1925 laut Mitteilung des Staatl. Hafenamtes Ludwigshafen. Basel: Rheinhäfen beider Basel, Lit. 24 und Zeitungsmeldung in "Neue Zürcher Zeitung" 18. I. 1960 Blatt $4-19$ Lit. 15 S. 66 und Lit. 21 ab $1947-20$ Lit. 20 Stat. Jahresbericht 1958 S. 109 113, Berufspendelwanderung: Einpendler auf Grund der Anmeldungen der Wohngemeinden für den Gewerbesteuerausgleich. - 21 Lit. 20 S. 61 - 22 Lit. 20 S. 114 - 23 Lit. 15 S. 69 - 24 Lit. 22 Stat. Jahresbericht 1957 - 25 Lit. 24 Jahrgang 1957 S. 255 - 26 Lit. 22 Stat. Jahresbericht 1958 - 27 Lit. 13 S.37.

\section{Schriflen}

\section{LITERATURVER Z E I CHN IS}

1 Barlet H.: Die Pendelwanderung im Rhein-Neckar-Raum. Diss. Mannheim 1953. Beiträge zur Statistik der Stadt Mannheim Heft 45 - 2 Bauverwaltung Ludwigshafen: Wirtschaftsplan 1956. Ludwigshafen 1956 - 3 Bauverwaltung Ludwigshafen: Gesamtverkehrsplan 1957, die Neuordnung der Innenstadt. Ludwigshafen 1957 - 4 Bundesministerium des Innern: Die Neugliederung des Bundesgebietes. Bonn 1955 - 5 Harrsen J. W'.: 350 Jahre Mannheim. Mannheim 1957 - 6 HooK K.: Mannheim in Wort, Zahl und Bild. Entwicklung seit 1900. Mannheim 1954 - 7 JАсов G.: Mannheim einst und jetzt. Mannheiın 1959 - 8 Institut zur Förderung öffentlicher Angelegenheiten: Die Bundesländer, Beiträge zur Neugliederung der Bundesrepublik. Wissenschaftliche Schriftenreihe Band 9. Frankfurt 1950 - 9 KLöPPER R.: Junge Industrie-Großstädte Ludwigshafen - Leverkusen Höchst. Festschrift Th. Kraus. Bad Godesberg 1959 - 10 KRÜGER H.-J.: Der Wirtschaftsraum Mannheim-Ludwigshafen. Diss. Mannheim 1955. Beiträge zur Statistik der Stadt Mannheim Heft 49 - 11 LÜвBEKE G.: Großfächige Eisenbahnplanung im Raume Mannheim - Ludwigshafen Heidelberg. Eisenbahntechnische Rundschau, Hefte 3 und 4. Darmstadt 1952 - 12 Plewe E.: Die Industrie von Mannheim - Ludwigshafen. Geogr. Rundschau Nr. 51954 - 13 PLewe E.: Zur Entwicklungsgeschichte der Stadt Mannheim. In Festschrift zur Einweihung der Wirschaftshochschule Mannheim im Mannheimer Schloß. Mannheim 1955 - 14 Sснотт S.: Ausgewählte Schriften. Beiträge zur Statistik der Stadt Mannheim Heft 52, 1957 - 15 Stadtverwaltung Ludwigshafen: Ludwigshafen, Stadt der Chemie. Hanau 1959 - 16 Walter F.: Aufgabe und Vermächtnis einer deutschen Stadt. Drei Jahrhunderte Alt-Mannheim. Frankfurt 1952.

\section{Zeitungen}

17 Ernährungsdienst (Deutsche Getreidezeitung): Beilage: Getreidezentrum Mannheim. 6.12.58.18 Handelsblatt (Deutsche Wirtschaftszeitung): Bilanz Deutscher Städte: Rhein-Neckarraum. 11.7.52.19 Rheinpfalz, Die (Ludwigshafener Tagblatt): Sonderbeilage $* 100$ Jahre Ludwigshafen $*$. 15. 4. 53. 


\title{
Statistiken
}

20 Statistisches Amt Mannheim: Mannheim, statistischer Jahresbericht - 21 Statistisches Amt Ludwigshafen: Wort und Zahl. Statistisches Monatsbulletin - 22 Hafenamt Mannheim, staatl.: Statistischer Jahresbericht über den Schiffs- und Güterverkehr in den Mannheimer Häfen einschl. Rheinau nebst vergleichenden Darstellungen - 23 Statistisches Amt Zürich: Statistisches Jahrbuch, 54. Jahrgang 1958 - 24 Eidgenössisches Statistisches Amt: Statistisches Jahrbuch der Schweiz.

Atlanten, Karten, Pläne

25 Ammann H.: Wirtschaft und Verkehr im Spätmittelalter um 1500. 1:3000 000. In Atlas "Oestliches Mitteleuropa», Blatt 14. Bad Godesberg 1959 - 26 Harms: Heimatatlas Mannheim. Frankfurt etwa 1958 - 27 Kögel B.: Harms Baden-Württemberg. Frankfurt etwa 1958 28 Meynen E. und Schmithüsen J.: Naturräumliche Gliederung Deutschlands, 1:1 000000 . In Literatur 4. Remagen 1954 - 29 RENARD R.: Harms Rheinland-Pfalz. Frankfurt etwa 1958 30 Städtisches Vermessungs- und Liegenschaftsamt Mannheim: Mannheim, 1:15000. Mannheim 1957.

\section{MAN N H E I M - LU DWIGSHAFEN}

Les deux villes, situées au centre de la Kurpfalz et par là soumises à des conditions commerciales et agricoles extrèmement favorables, ont subi de nombreuses inondations pendant leur histoire. Le développement de Mannheim était néanmoins très rapide et s'accomplissait en plusieurs stades: village de pêcheurs, ville fortifiée, Résidence de la Kurpfalz, point final de la navigation fluviale, centre commercial, ville industrielle, port d'une importance de plus en plus croissante. Ludwigshafen, une simple bastion au 18ième siècle, devenait un centre de produits chimiques de la plus grande importance. Près de l'embouchure du Neckar s'est formé ainsi une des régions les plus industrialisées de l'Allemagne occidentale du sud, dont le manque d'intégrité politique empêche malheureusement souvent les efforts entrepris pour un développement économique universel.

\section{DIE GEOMORPHOLOGISCHEN KRÄFTE DER GEGENWART}

\author{
EINE GEOGRAPHENTAGUNG IN BASEL
}

Anläßlich des Wechsels im Vorort veranstaltete der Verband Schweiz. Geographischer Gesellschaften zusammen mit der Schweiz. Geomorphologischen Gesellschaft am 7./8. November 1959 in Basel, in einem der Auditorien der Universität, eine wissenschaftliche Geographentagung, der zahlreiche Teilnehmer aus dem In- und Ausland Folge leisteten. Das Thema galt der Wirkung der morphologischen Kräfte in der Gegenwart und ihrem Anteil an der Gestaltung des Formenschatzes der Erdoberfläche.

In der einleitenden administrativen Sitzung wies der Vorsitzende, Dr. E. Schwabe (Bern), u. a. auf die Vorarbeiten für den in den nächsten Jahren zu erstellenden schweizerischen Landesatlas hin, die unter der tatkräftigen Mitwirkung von Prof. Dr. E. ImHoF haben gefördert werden können. Nach den verschiedenen Berichterstattungen, so von Seiten der einzelnen Verbandsgesellschaften, welche deren allgemeine Aufwärtsentwicklung erkennen ließen, erfolgte die Übergabe des Verbandsvorortes von der Schweiz. Geomorphologischen Gesellschaft (bisheriger Zentralpräsident Dr. ScHWABE) an die Geographisch Ethnographische Gesellschaft Zürich, mit Prof. Dr. H. Gutersohn als Zentralpräsident, Prof. Dr. E. WinkLer als Vizepräsident und Sekretär und A. SchäpPI als Quästor.

Die wissenschaftliche Versammlung wurde am Samstag-Nachmittag mit Begrüßungsworten von Dr. E. Schwabe im Namen der Organisatoren und von Prof. Dr. P. Vosseler im Namen der Universität Basel und der Basler Geographen eröffnet. Prof. Dr. H. BoEsch (Zürich) betonte anschließend die Bedeutung der Antarktisforschung für das Studium der pleistozänen Verhältnisse in Mitteleuropa. Er wies dabei, außer auf die übereinstimmenden Erscheinungen, auch auf wichtige Unterschiede, besonders auf klimatischem Gebiet, hin, welche zwischen der Arktis und den gemäßigten Breiten bestehen.

Prof. Dr. J. Büdel (Würzburg) griff in ausführlicherer Weise ähnliche Probleme auf, indem er, an Beispielen aus dem deutschen Mittelgebirge und von den Inselbergen in den nord- 